\title{
The addicted human brain: insights from imaging studies
}

\author{
Nora D. Volkow, ${ }^{1,2}$ Joanna S. Fowler, ${ }^{3}$ and Gene-Jack Wang ${ }^{1}$ \\ ${ }^{1}$ Department of Medicine, Brookhaven National Laboratory, Upton, New York, USA \\ ${ }^{2}$ Department of Psychiatry, State University of New York at Stony Brook, Stony Brook, New York, USA \\ ${ }^{3}$ Department of Chemistry, Brookhaven National Laboratory, Upton, New York, USA \\ J. Clin. Invest. 111:1444-1451 (2003). doi:10.1172/JCI200318533.
}

Imaging studies have revealed neurochemical and functional changes in the brains of drug-addicted subjects that provide new insights into the mechanisms underlying addiction. Neurochemical studies have shown that large and fast increases in dopamine are associated with the reinforcing effects of drugs of abuse, but also that after chronic drug abuse and during withdrawal, brain dopamine function is markedly decreased and these decreases are associated with dysfunction of prefrontal regions (including orbitofrontal cortex and cingulate gyrus). The changes in brain dopamine function are likely to result in decreased sensitivity to natural reinforcers since dopamine also mediates the reinforcing effects of natural reinforcers and on disruption of frontal cortical functions, such as inhibitory control and salience attribution. Functional imaging studies have shown that during drug intoxication, or during craving, these frontal regions become activated as part of a complex pattern that includes brain circuits involved with reward (nucleus accumbens), motivation (orbitofrontal cortex), memory (amygdala and hippocampus), and cognitive control (prefrontal cortex and cingulate gyrus). Here, we integrate these findings and propose a model that attempts to explain the loss of control and compulsive drug intake that characterize addiction. Specifically, we propose that in drug addiction the value of the drug and drug-related stimuli is enhanced at the expense of other reinforcers. This is a consequence of conditioned learning and of the resetting of reward thresholds as an adaptation to the high levels of stimulation induced by drugs of abuse. In this model, during exposure to the drug or drug-related cues, the memory of the expected reward results in overactivation

\footnotetext{
Address correspondence to: Nora D. Volkow, Department of Medicine, Brookhaven National Laboratory, Upton, New York 11973, USA. Phone: (631) 344-3335; Fax: (631) 344-5260; E-mail: volkow@bnl.gov.

Conflict of interest: The authors have declared that no conflict of interest exists.

Nonstandard abbreviations used: positron emission tomography (PET); functional magnetic resonance imaging (fMRI); dopamine (DA); nucleus accumbens (NAc); orbitofrontal cortex (OFC); cingulate gyrus (CG).
}

of the reward and motivation circuits while decreasing the activity in the cognitive control circuit. This contributes to an inability to inhibit the drive to seek and consume the drug and results in compulsive drug intake. This model has implications for therapy, for it suggests a multi-prong approach that targets strategies to decrease the rewarding properties of drugs, to enhance the rewarding properties of alternative reinforcers, to interfere with conditioned-learned associations, and to strengthen cognitive control in the treatment of drug addiction.

\section{Introduction}

Addiction is a disorder that involves complex interactions between biological and environmental variables (1). This has made treatment particularly elusive, since attempts to categorize addiction have usually concentrated on one level of analysis. Attempts to understand and treat addiction as a purely biological or a purely environmental problem have not been very successful. Recently, important discoveries have increased our knowledge about how drugs of abuse affect biological factors such as genes, protein expression, and neuronal circuits $(2,3)$; however, much less is known about how these biological factors affect human behavior. Nor do we know much about how environmental factors affect these biological factors and how these in turn alter behavior. Relatively new imaging technologies such as positron emission tomography (PET) and functional magnetic resonance imaging (fMRI) have provided new ways to investigate how the biological factors integrate with one another, how they relate to behavior, and how biological and environmental variables interact in drug addiction (Figure 1).

PET imaging is based on the use of radiotracers labeled with short-lived positron-emitting isotopes (carbon-11, oxygen-15, nitrogen-13, and fluorine-18), which it can measure at very low concentrations (nanomolar to picomolar range) (4). Therefore, PET can be used to measure labeled compounds that selectively bind to specific receptors, transporters, or enzyme types at concentrations that do not perturb function (Figure 2). fMRI is based on the measurement 


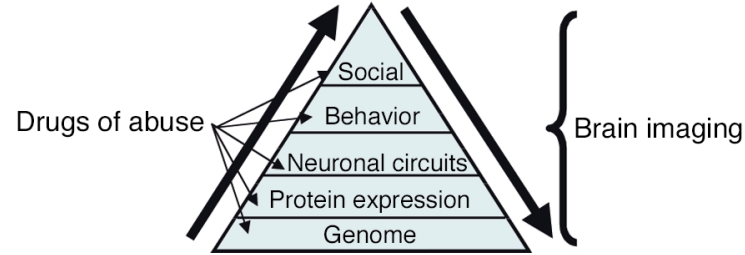

\section{Figure 1}

Drugs of abuse have effects at multiple biological and environmental levels. The environmental level is identified as "social," since this is the most relevant of the environmental factors that influence drug abuse in humans. Imaging techniques allow one to assess the effects of drugs of abuse at the protein and the brain circuit levels and to assess how these effects relate to behavior. Imaging also offers a way to start to assess the impact of environmental factors on these biological levels, as well as the impact of gene polymorphisms on protein expression and brain function.

of the changes in magnetic properties in neuronal tissue (4). It is generally believed that the activation signal generated from $\mathrm{AMRI}$ results from differences in the magnetic properties of oxygenated versus deoxygenated hemoglobin (blood oxygen level dependent contrast). During activation of a brain region there is an excess of arterial blood delivered into the area, with concomitant changes in the ratio of deoxyhemoglobin to oxyhemoglobin.

Most PET studies of drug addiction have concentrated on the brain dopamine (DA) system, since this is considered to be the neurotransmitter system through which most drugs of abuse exert their reinforcing effects (5). A reinforcer is operationally defined as an event that increases the probability of a subsequent response, and drugs of abuse are considered to be much stronger reinforcers than natural reinforcers (e.g., sex and food) (6).The brain DA system also regulates motivation and drive for everyday activities (7). These imaging studies have revealed that acute and chronic drug consumption have different effects on proteins involved in DA synaptic transmission (Figure 2). Whereas acute drug administration increases DA neurotransmission, chronic drug consumption results in a marked decrease in DA activity, which persists months after detoxification and which is associated with deregulation of frontal brain regions (8). PET and MRI studies have characterized the brain areas and circuits involved in various states of the drug addiction process (intoxication, withdrawal, and craving) and have linked the activity in these neural circuits to behavior (Figure 3). Acute drug intoxication results in a complex and dynamic pattern of activation and deactivation that includes regions neuroanatomically connected with the DA system and known to be involved in reward, memory, motivation/drive, and control $(9,10)$. The same imaging methods have been used to demonstrate how environmental factors can influence these neuronal circuits, which in turn affect behavior related to drug addiction (e.g., drug consumption). For example, a recent study in nonhuman primates showed that social status affects DA D2 receptor expression in the brain, which in turn affects the propensity for cocaine self-administration (11) (Figure 4).

Here we analyze the results from our imaging program in drug addiction, and from the rich literature, and integrate this body of knowledge with preclinical findings to develop a model that could explain the loss of control and compulsive drug intake observed in the addicted individual.

\section{Drug addiction involves multiple brain circuits}

The aforementioned model proposes a network of four circuits involved in drug abuse and addiction: (a) reward, located in the nucleus accumbens (NAc) and the ventral pallidum; (b) motivation/drive, located in the orbitofrontal cortex (OFC) and the subcallosal cortex; (c) memory and learning, located in the amygdala and the hippocampus; and (d) control, located in the prefrontal cortex and the anterior cingulate gyrus (CG) (Figure 5). These four circuits receive direct innervations from DA neurons but are also connected with one another through direct or indirect projections (mostly glutamatergic). Though we have identified specific brain regions associated with each circuit, we have realized that other brain regions are involved in these circuits (e.g., the thalamus and insula), that one region may participate in more than one circuit (e.g., the CG in both control and motivation/drive circuits), and that other brain

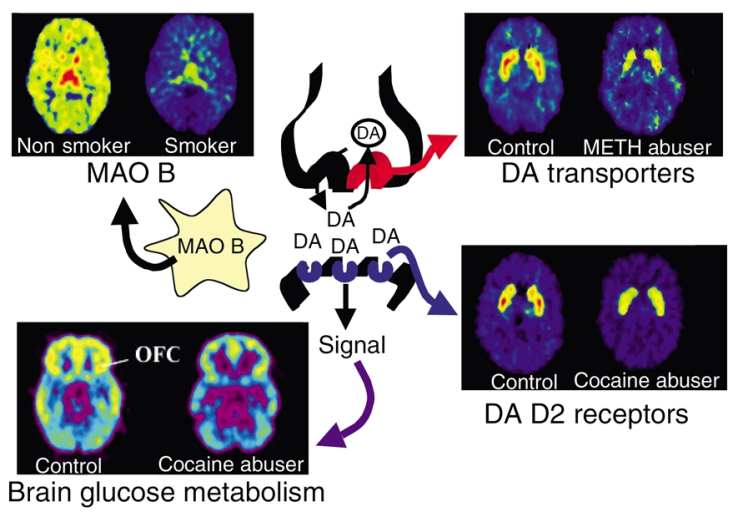

Figure 2

Images obtained with PET (axial sections) that show the effects of chronic drug exposure on various proteins involved in dopamine (DA) neurotransmission and on brain function (as assessed by brain glucose metabolism). While some effects are common to many drugs of abuse, such as decreases in DA D2 receptors in striatal neurons and decreased metabolic activity in the orbitofrontal cortex (OFC), others are more specific. These include the decrease in DA transporters in striatum in methamphetamine (METH) abusers (possibly the result of neurotoxicity to DA terminals) and the decrease in brain monoamine oxidase $\mathrm{B}$ (MAO B; the enzyme involved in DA metabolism) in cigarette smokers. The rainbow scale was used to code the PET images; radiotracer concentration is displayed from higher to lower as red > yellow > green > blue. Images from methamphetamine use are adapted from ref. 61. Images from smokers are adapted with permission from ref. 62 . 


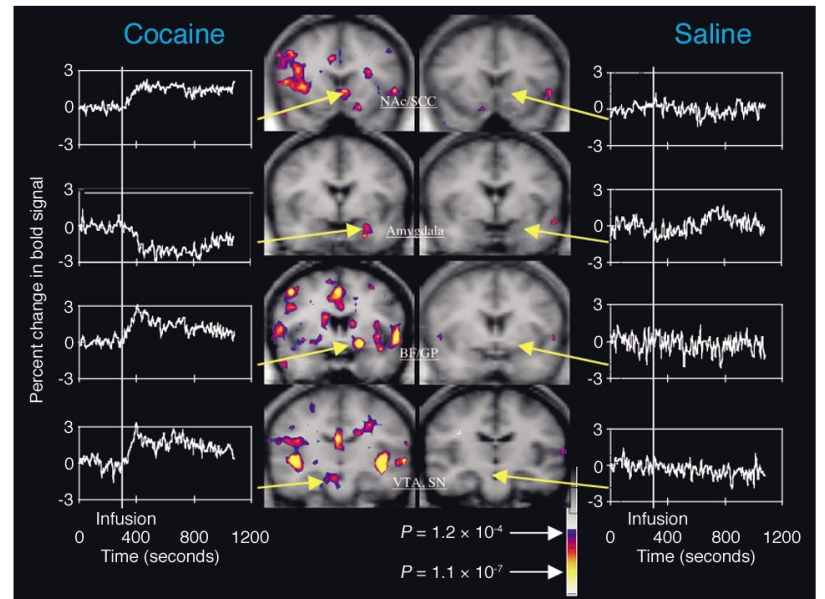

Figure 3

Images of coronal sections obtained with $\mathrm{AMRI}$, showing areas of brain activation and deactivation during cocaine intoxication compared with those after saline administration. During intoxication there is a complex pattern of activation and/or deactivation that includes the ventral tegmental area (VTA) and the substantia nigra $(\mathrm{SN})$, where DA cells are located, as well as regions involved with reward (nucleus accumbens, NAc; basal forebrain, BF; globus pallidus, GP), with memory (amygdala), and with motivation (subcallosal cortex, SCC). The color scale indicates the level of significance ( $P$ value) of the change in activation of the bold signal. Reproduced with permission from Neuron (9).

regions (e.g., the cerebellum) and circuits (e.g., attention and emotion circuits) are likely to be affected in drug addiction. Though our model focuses on DA, it is evident from preclinical studies that modifications in glutamatergic projections mediate many of the adaptations observed with addiction (12). Unfortunately, the lack of radiotracers available to image glutamate function in the human brain has precluded its investigation in drug-addicted subjects.

We propose that the pattern of activity in the fourcircuit network outlined in Figure 5 influences how an individual makes choices among behavioral alternatives. These choices are influenced systematically by the reward, memory, motivation, and control circuits. The response to a stimulus is affected by its momentary saliency - i.e., expected reward, which is processed in part by DA neurons projecting into the NAc (13) - in a hierarchical matrix where the saliency value of stimuli changes as a function of the context and the previous experience of the individual. If the individual has been previously exposed to the stimulus, its saliency value is affected by memory, processed in part by the amygdala and hippocampus. Memories are stored as associations between the stimulus and the positive (pleasant) or negative (aversive) experience it elicited and are facilitated by DA activation (14). The value of the stimulus is weighted against that of other alternative stimuli and changes as a function of the internal needs of the individual, which are processed in part by the $\operatorname{OFC}(15,16)$. For example, the saliency value of food is increased by hunger but decreased by satiety. The stronger the saliency value of the stimulus, which is in part conveyed by the prediction of reward from previously memorized experiences, the greater the activation of the motivational circuit and the stronger the drive to procure it. The cognitive decision to act (or not) to procure the stimulus is processed in part by the prefrontal cortex and the CG (17).

The model proposes that, in the addicted subject, the saliency value of the drug of abuse and its associated cues is enhanced in the reward and motivation/drive circuits but that of other reinforcers is markedly decreased. The enhanced saliency value of the drug of abuse is initiated partly by the much higher intrinsic reward properties of drugs of abuse: increases in DA induced by drugs in the NAc are three- to fivefold higher than those of natural reinforcers (7). Another cause of the enhanced saliency is the lack of habituation of drugs of abuse as compared with that of natural reinforcers (18). It is postulated that the high reward value of drugs leads to a resetting of reward thresholds, which then results in decreased sensitivity to the reinforcing properties of naturally occurring stimuli (19). Through conditioned learning and a lack of competition by other reinforcers, acquisition of the drug becomes the main motivational drive for the individual. We hypothesize that, during intoxication, the qualitative difference in activity in the DA-regulated reward circuit (greater and longer-lasting activation compared with the activation by nondrug stimuli) (18) produces a corresponding overactivation of the motivational/drive
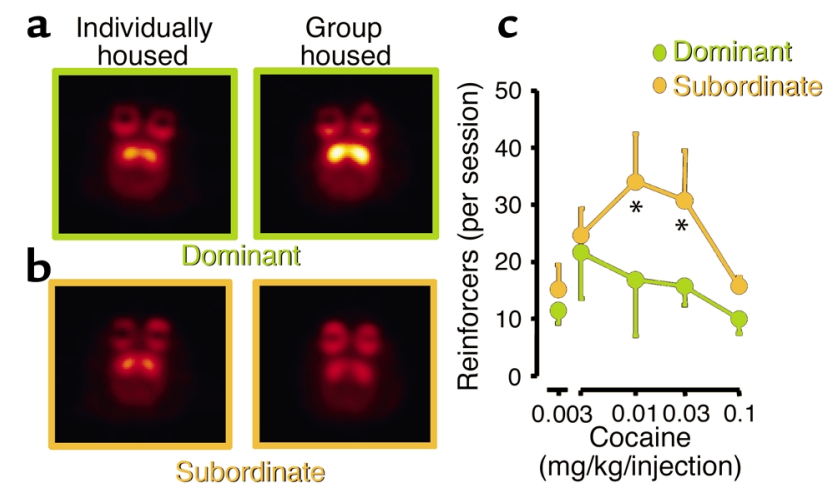

Figure 4

Images of axial sections obtained with PET, showing DA D2 receptors in nonhuman primates that were initially tested while housed in separate cages and then retested after being housed in a group. Animals that became dominant when placed in a group (a) showed increased numbers of DA D2 receptors in striatum, whereas subordinate animals (b) did not. (c) The levels of cocaine administration in the subordinate and the dominant animals. Note the much lower intake of cocaine by dominant animals which possessed higher numbers of DA D2 receptors. The temperature scale was used to code the PET images; radiotracer concentration is displayed from higher to lower as yellow $>$ red. Asterisks indicate significant differences in drug intake between groups. Adapted with permission from ref. 11. 

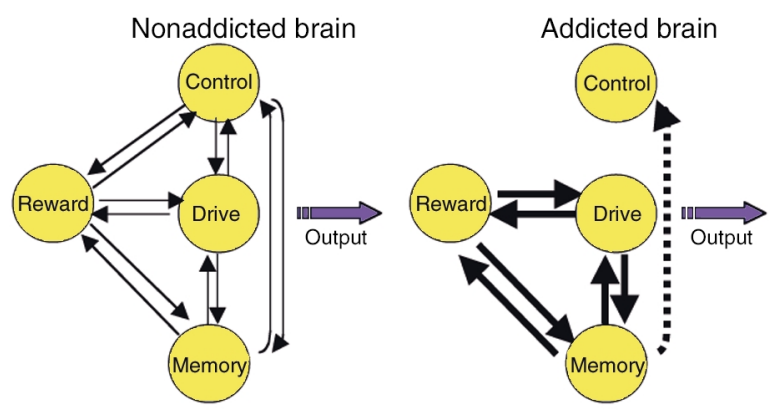

Figure 5

Model proposing a network of four circuits involved with addiction: reward, motivation/drive, memory, and control. These circuits work together and change with experience. Each is linked to an important concept: saliency (reward), internal state (motivation/drive), learned associations (memory), and conflict resolution (control). During addiction, the enhanced value of the drug in the reward, motivation, and memory circuits overcomes the inhibitory control exerted by the prefrontal cortex, thereby favoring a positive-feedback loop initiated by the consumption of the drug and perpetuated by the enhanced activation of the motivation/drive and memory circuits.

and memory circuits, which deactivate and remove the control exerted by the frontal cortex. Without the inhibitory control, a positive-feedback loop is set forth that results in compulsive drug intake (Figure $5)$. Because the interactions between the circuits are bidirectional, the activation of the network during intoxication serves to further strengthen the saliency value of the drug.

\section{Reward circuit in drug addiction}

The reinforcing effects of drugs during intoxication create an environment that, if perpetuated, triggers the neuronal adaptations that result in addiction. Imaging studies in drug abusers as well as non-drug abusers have shown that drugs of abuse increase the extracellular concentration of DA in the striatum (where the NAc is located) and that these increases were associated with their reinforcing effects. The subjects who had the greatest increases in DA were the ones who experienced drug effects such as "high," "rush," or "euphoria" most intensely (20-22). These studies also showed that the reinforcing effects appeared to be associated not only with the magnitude but also with the abruptness of the DA increase. Thus, for an equivalent increase in DA, the drug was experienced as reinforcing when it was injected intravenously (21), which leads to fast drug uptake in the brain and presumably very fast changes in DA concentration, but not when it was given orally (23), which leads to a slow rate of brain uptake and presumably slow increases in DA concentration. The dependency of the reinforcing effects of drugs on fast and large increases in DA concentration is reminiscent of the changes in DA concentration induced by phasic DA cell firing (fast-burst firing > $30 \mathrm{~Hz})(6)$, which also leads to fast changes in DA concentration and whose function is to highlight the saliency of stimuli (24). This contrasts with tonic DA cell firing (slow firing at frequencies around $5 \mathrm{~Hz}$ ) (6), which maintains base-line steady-state DA levels and whose function is to set the overall responsiveness of the DA system. This led us to speculate that the ability of drugs of abuse to induce changes in DA concentration that mimic but exceed those produced by phasic DA cell firing results in overactivation of the neuronal processes that highlight saliency, and that this is one of the relevant variables underlying their high reinforcing value.

However, studies show that increases in DA concentration during intoxication occur in both addicted and nonaddicted subjects, so this by itself cannot explain the process of addiction. Since drug addiction requires chronic drug administration, we suggest that addiction results from the repeated perturbation of reward circuits (marked DA increases followed by DA decreases) and the consequent disruption of the circuits that it regulates (motivation/drive, memory/learning, and control). Indeed, imaging studies in drug-addicted subjects have consistently shown long-lasting decreases in the numbers of DA D2 receptors in drug abusers compared with controls (Figure 2) (reviewed in ref. 8). In addition, studies have shown that cocaine abusers also have decreased DA cell activity, as evidenced by reduced DA release in response to a pharmacological challenge with a stimulant drug (25). We postulate that the decrease in the number of DA D2 receptors, coupled with the decrease in DA cell activity, in the drug abusers would result in a decreased sensitivity of reward circuits to stimulation by natural reinforcers. This decreased sensitivity would lead to decreased interest in ordinary (day-to-day) environmental stimuli, possibly predisposing subjects for seeking drug stimulation as a means to temporarily activate these reward circuits. Imaging studies provide

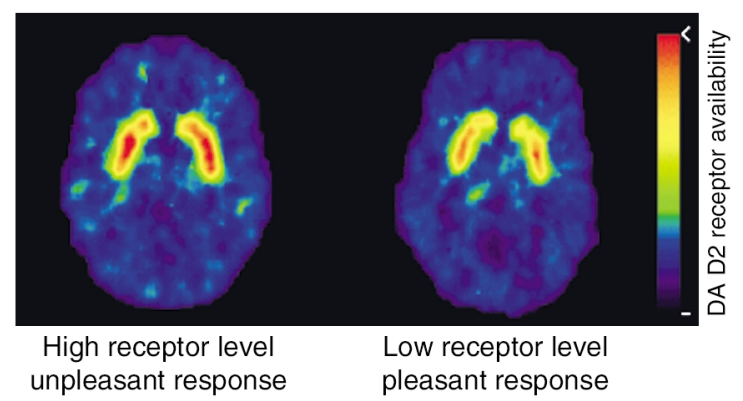

\section{Figure 6}

Images of axial sections obtained with PET to measure the numbers of DA D2 receptors in subjects who reported the effects of the stimulant drug methylphenidate as pleasant versus those that reported its effects as unpleasant. Subjects with high numbers of DA D2 receptors tended to report the effects of methylphenidate as unpleasant, whereas subjects with low numbers of DA D2 receptors tended to report it as pleasant. The rainbow scale was used to code the PET images; radiotracer concentration is displayed from higher to lower as red $>$ yellow $>$ green $>$ blue. Adapted with permission from ref. 53 . 
evidence of disrupted sensitivity to natural reinforcers in addiction. For example, in a study by Martin-Solch and colleagues (25), the meso-striatal and meso-corticolimbic circuits of opiate addicts were not activated in response to natural reinforcers, whereas they were in controls subjects. Similarly, in a second study by the same group, DA-regulated reward centers in tobacco smokers failed to activate in response to monetary reward (26). Interestingly, decreased sensitivity of reward circuits to acute alcohol administration has also been documented in cocaine abusers compared with control subjects (27). These findings suggest an overall reduction in the sensitivity of reward circuits in drug-addicted individuals to natural reinforcers, but also possibly to drugs besides the one to which they are addicted.

\section{Motivation/drive circuit in addiction}

We postulate that, during addiction, the value of the drug as a reinforcer is so much greater than that of any natural reinforcer that these can no longer compete as viable alternative choices, and the enhanced saliency value of the drug becomes fixed. This contrasts with natural reinforcers, whose saliency is momentary and decreases with exposure to the reinforcer (18) or with the presentation of an alternative, more appealing reinforcer. One area of the brain that is involved in shifting the relative value of reinforcers is the $\operatorname{OFC}(15,16)$.

Imaging studies have provided evidence of disruption of the OFC during addiction (reviewed in ref. 28) (Figure 2). The OFC appears to be hypoactive in drugaddicted subjects tested during withdrawal $(29,30)$; we postulate that this results from the lack of stimulation by salient stimuli during detoxification. In contrast, in active cocaine abusers, the OFC has been shown to be hypermetabolic in proportion to the intensity of the craving experienced by the subjects (31). We therefore postulated that exposure to the drug or drug-related stimuli in the withdrawal state reactivates the OFC and results in compulsive drug intake. Indeed, activation of the OFC has been reported during drug intoxication in drug-addicted, but not in non-drug-addicted, subjects, and the level of activation predicted the intensity of drug-induced craving $(32,33)$. Similarly, activation of the OFC has been reported during exposure to drug-related cues when these elicit craving (reviewed in ref. 28). Since increased OFC activation has been associated with compulsive disorders (reviewed in ref. 34), we postulated that the activation of the OFC in addicted subjects contributes to the compulsive drug intake. Indeed, preclinical studies have shown that damage of the OFC results in a behavioral compulsion to procure the reward even when it is no longer reinforcing (16). This is consistent with the accounts of drug addicts who claim that once they start taking the drug they cannot stop, even when the drug is no longer pleasurable. Since the OFC also processes information associated with the prediction of reward (15), its activation during cue exposure could signal reward prediction, which could then be experienced as craving by the addicted subject.

In detoxified drug abusers, the decreased activity in the OFC is associated with reductions in the numbers of DA $\mathrm{D} 2$ receptors in striatum $(35,36)$. Since DA D2 receptors transmit reward signals into the OFC, this association could be interpreted as a disruption of the OFC, secondary to changes in striatal DA activity (such as lack of stimulation during withdrawal and enhanced stimulation with exposure to drugs or drug-related cues). However, since striatal-frontal connections are bidirectional, this association could also reflect the disruption of the OFC, which then deregulates DA cell activity.

\section{Learning/memory circuit in addiction}

The relevance of learning and memory to addiction is made evident by the pernicious effect that a place, a person, or a cue that brings back memories of the drug can have on the addict who is trying to stay clean. These factors trigger an intense desire for the drug (a craving) and, not infrequently, relapse. Multiple memory systems have been proposed in drug addiction, including conditioned-incentive learning (mediated in part by the NAc and the amygdala), habit learning (mediated in part by the caudate and the putamen), and declarative memory (mediated in part by the hippocampus) (reviewed in ref. 37). Through conditioned-incentive learning, the neutral stimuli, coupled with the drug of abuse, acquire reinforcing properties and motivational salience even in the absence of the drug. Through habit learning, well-learned sequences of behavior are elicited automatically by the appropriate stimuli. Finally, declarative memory is related to the learning of affective states in relationship to drug intake.

Memory circuits are likely to influence the effects of the drug during intoxication, since they set the expectations of the drug's effects in the addicted subject (38). Activation of regions linked with memory has been reported during drug intoxication $(9,10)$ and during craving induced by drug exposure, video, or recall (39-42). Also, studies in drug abusers during withdrawal have shown evidence of decreased D2 receptor expression and decreased DA release in the dorsal striatum (25). In animal studies, the drug-induced changes in the dorsal striatum are observed after longer drug exposures than those observed in the NAc and have been interpreted to reflect further progression into the addicted state (43). This is relevant because involvement of the dorsal striatum, which is a region associated with habit learning, indicates that in drug addiction the routine associated with drug consumption may be triggered automatically by exposure to the drug or drug-related cues (44).

\section{Control circuit in addiction}

One of the most consistent findings from imaging studies is that of abnormalities in the prefrontal cortex, including the anterior CG, in drug-addicted subjects 
(reviewed in ref. 45). The prefrontal cortex is involved in decision making and in inhibitory control (reviewed in ref. 46). Thus its disruption could lead to inadequate decisions that favor immediate rewards over delayed but more favorable responses. It could also account for the impaired control over the intake of the drug even when the addicted subject expresses the desire to refrain from taking the drug (45). Thus, one might expect that the disruptions of self-monitoring and decision-making processes that are observed in drugaddicted subjects $(47,48)$ are in part related to disrupted prefrontal functions. Moreover, preclinical studies show that chronic administration of cocaine or amphetamine results in a significant increase in dendritic branching and the density of dendritic spines in the prefrontal cortex (49). These changes in synaptic connectivity could be involved in the changes in decision making, judgment, and cognitive control that occur during addiction. Indeed, imaging studies have shown evidence of changes in prefrontal activation during a working-memory task in smokers compared with ex-smokers (50).

We propose that disruption of the prefrontal cortex could lead to loss of self-directed/willed behavior in favor of automatic sensory-driven behavior (45). Moreover, the disruption of self-controlled behavior is likely to be exacerbated during drug intoxication from the loss of inhibitory control that the prefrontal cortex exerts over the amygdala (51). The inhibition of topdown control would release behaviors that are normally kept under close monitoring and would simulate stress-like reactions in which control is inhibited and stimulus-driven behavior is facilitated (45).

\section{Vulnerability to drug addiction}

A challenging problem in the neurobiology of drug addiction is to understand why some individuals become addicted to drugs while others do not. The model we propose offers some guidance as to specific disruptions that could make a subject more or less vulnerable to addiction. For example, one could hypothesize that decreased sensitivity of reward circuits to natural reinforcers, decreased activity of control circuits, or an increased sensitivity of memory/ learning or motivation/drive circuits to drug or drugrelated stimuli could make an individual more vulnerable to addiction.

In fact, imaging studies have provided evidence that differences in reward circuits may be one of the mechanisms underlying the variability in responsiveness to drugs of abuse, which in turn could influence vulnerability. These studies assessed the extent to which the variability in the number of DA D2 receptors in non-drug-abusing subjects affected their sensitivity to stimulant drugs (52). The data showed that subjects with low numbers of DA D2 receptors tended to describe the effects of the stimulant drug methylphenidate as pleasant, whereas subjects with high numbers of DA D2 receptors tended to describe it as unpleasant (Figure 6). Another study documented that the numbers of DA D2 receptors predicted how much subjects liked the effects of methylphenidate (53). These findings suggest that one of the mechanisms underlying the differences between subjects in their vulnerability to stimulant abuse may be the variability in the expression of DA D2 receptors. Subjects with low numbers of $\mathrm{D} 2$ receptors may be at higher risk of abusing stimulant drugs than those with high numbers of D2 receptors, in whom drugs such as methylphenidate may produce unpleasant effects that limit its abuse. A causal association between DA D2 receptor numbers and propensity to self-administer drugs was corroborated by a parallel preclinical study that showed that insertion of the DA D2 receptor gene via a viral vector to increase DA D2 receptor expression in the NAc of rats previously trained to self-administer alcohol resulted in marked reductions in alcohol intake (54). Alcohol intake recovered as the number of DA D2 receptors returned to baseline levels. These results could be taken as indirect evidence of a protective role of high DA D2 receptor numbers against drug abuse. Baseline levels of DA D2 receptors in the brain, which have been shown to be affected by stress (55) and social hierarchy (11), provide a molecular mechanism that could explain the influence of the environment and genetics on predisposition to drug abuse.

Recently, imaging studies showed that offspring of alcoholic families who were considered to be at high risk for alcoholism showed smaller amygdala volumes in comparison with control subjects (56). Moreover, the volume of the amygdala was associated with the amplitude of the P300 in the evoked potential (wave occurring between 300 and $500 \mathrm{~ms}$ after a rare target stimulus), which is considered to be a phenotypic marker for vulnerability to alcoholism. Also, a recent imaging study reported structural changes in the OFC of cocaine-addicted subjects (57), and the possibility was discussed that this might have preceded drug use and might have made these subjects more vulnerable to addiction.

Access to transgenic and knockout animals now provides a means to directly evaluate the role that specific genes may play in vulnerability to, or protection against, drug abuse and addiction (58). Thus, information from imaging studies regarding abnormalities in specific proteins in the brains of drugaddicted subjects (e.g., DA D2 receptors and monoamine oxidase B) can now be tested in preclinical models to determine whether these abnormalities reflect changes that preceded drug use and are genetically determined, or whether they are a consequence of chronic drug use.

\section{Conclusion}

Here we provide a model that conceptualizes addiction as a state initiated by the qualitatively different and larger reward value of the drug, which triggers a 
series of adaptations in the reward, motivation/drive, memory, and control circuits of the brain. These changes result in an enhanced and permanent saliency value for the drug, and in the loss of inhibitory control, favoring the emergence of compulsive drug administration. The model has treatment implications, for it suggests strategies to combat drug addiction - specifically (a) interventions to decrease the rewarding value of drugs, such as pharmacological treatments that interfere with the drug's reinforcing effects as well as treatments that make the effects unpleasant; (b) interventions to increase the value of nondrug reinforcers, such as pharmacological and behavioral treatments that increase sensitivity to natural reinforcers and establish alternative reinforcing behaviors; (c) interventions to weaken learned drug responses, such as behavioral treatments to extinguish the learned positive associations with the drug and drug cues but also promote differential reinforcement of other behaviors; and (d) interventions to strengthen frontal control, such as cognitive therapy. The model also highlights the need for therapeutic approaches that include pharmacological as well as behavioral interventions in the treatment of drug addiction (59).

This analysis brings to light the paucity of PET radiotracers currently available for use in imaging of the human brain. Further research on the development of radiotracers that can be used to target other neurotransmitter systems affected by drugs of abuse (e.g., glutamate and $\gamma$-aminobutyric acid) will in the future provide a more complete picture of the neurochemical changes that underlie drug addiction. Moreover, access to a wider array of radiotracers will enable researchers to start to investigate the role that gene polymorphisms may play in protein expression, and how this in turn relates to behavioral responses to drugs of abuse (60).

\section{Acknowledgments}

The authors are indebted to the Department of Energy (Office of Biological and Environmental Research; DE-ACO2-98CH10886), the National Institute on Drug Abuse (DA-06278, DA-09490, and DA-06891), the National Institute on Alcohol Abuse and Alcoholism (AA/OD-09481), and the Office of National Drug Control Policy for support of our research. We are also indebted to our scientific and technical colleagues and our research volunteers, without whom our efforts on drug addiction would not have been able to proceed.

1. Leshner, A.I. 1997. Addiction is a brain disease, and it matters. Science. 278:45-47.

2. Nestler, E.J. 2001. Molecular basis of long-term plasticity underlying addiction. Nat. Rev. Neurosci. 2:119-128.

3. Hyman, S.E., and Malenka, R.C. 2001. Addiction and the brain: the neurobiology of compulsion and its persistence. Nat. Rev. Neurosci. 2:695-703.

4. Volkow, N.D., Rosen, B., and Farde, L. 1997. Imaging the living human brain: magnetic resonance imaging and positron emission tomography. Proc. Natl. Acad. Sci. U. S. A. 94:2787-2788.
5. Koob, G.F., and Bloom, F.E. 1988. Cellular and molecular mechanism of drug dependence. Science. 242:715-723.

6. Wightman, R.M., and Robinson, D.L. 2002. Transient changes in mesolimbic dopamine and their association with 'reward'. J. Neurochem. 82:721-735.

7. Wise, R.A. 2002. Brain reward circuitry: insights from unsensed incentives. Neuron. 36:229-240.

8. Volkow, N.D., Fowler, J.S., and Wang, G.J. 2002. Role of dopamine in drug reinforcement and addiction in humans: results from imaging studies. Behav. Pharmacol. 13:355-366.

9. Breiter, H.C., et al. 1997. Acute effects of cocaine on human brain activity and emotion. Neuron. 19:591-611.

10. Stein, E.A., et al. 1998. Nicotine-induced limbic cortical activation in the human brain: a functional MRI study. Am. J. Psychiatry. 155:1009-1015.

11. Morgan, D., et al. 2002. Social dominance in monkeys: dopamine D2 receptors and cocaine self-administration. Nat. Neurosci. 5:169-174.

12. Cornish, J.L., and Kalivas, P.W. 2001. Cocaine sensitization and craving: differing roles for dopamine and glutamate in the nucleus accumbens. J. Addict. Dis. 20:43-54.

13. Dehaene, S., and Changeux, J.P. 2000. Reward-dependent learning in neuronal networks for planning and decision making. Prog. Brain Res. 126:217-229.

14. Di Chiara, G. 1999. Drug addiction as dopamine-dependent associative learning disorder. Eur. J. Pharmacol. 375:13-30.

15. Schultz, W., Tremblay, L., and Hollerman, J.R. 2000. Reward processing in primate orbitofrontal cortex and basal ganglia. Cereb. Cortex. 10:272-284.

16. Rolls, E.T. 2000. The orbitofrontal cortex and reward. Cereb. Cortex. 10:284-294.

17. Miller, E.K., and Cohen, J.D. 2001. An integrative theory of prefrontal cortex function. Annu. Rev. Neurosci. 24:167-202.

18. Di Chiara, G. 2002. Nucleus accumbens shell and core dopamine: differential role in behavior and addiction. Behav. Brain Res. 137:75-114.

19. Koob, G.F., and Le Moal, M. 2001. Drug addiction, dysregulation of reward, and allostasis. Neuropsychopharmacology. 24:97-129.

20. Laruelle, M., et al. 1995. SPECT imaging of striatal dopamine release after amphetamine challenge. J. Nucl. Med. 36:1182-1190.

21. Volkow, N.D., et al. 1999. Reinforcing effects of psychostimulants in humans are associated with increases in brain dopamine and occupancy of D(2) receptors. J. Pharmacol. Exp. Ther. 291:409-415.

22. Drevets, W.C., et al. 2001. Amphetamine-induced dopamine release in human ventral striatum correlates with euphoria. Biol. Psychiatry. 49:81-96.

23. Volkow, N.D., et al. 2001. Therapeutic doses of oral methylphenidate significantly increase extracellular dopamine in the human brain. J. Neurosci. 21:RC121.

24. Grace, A.A. 2000. The tonic/phasic model of dopamine system regulation and its implications for understanding alcohol and psychostimulant craving. Addiction. 95:S119-S128.

25. Volkow, N.D., et al. 1997. Decreased striatal dopaminergic responsivity in detoxified cocaine abusers. Nature. 386:830-833.

26. Martin-Solch, C., et al. 2001. Changes in brain activation associated with reward processing in smokers and nonsmokers. A positron emission tomography study. Exp. Brain Res. 139:278-286.

27. Volkow, N.D., et al. 2000. Cocaine abusers show a blunted response to alcohol intoxication in limbic brain regions. Life Sci. 66:PL161-PL167.

28. Volkow, N.D., and Fowler, J.S. 2000. Addiction, a disease of compulsion and drive: involvement of the orbitofrontal cortex. Cereb. Cortex. 10:318-325.

29. Volkow, N.D., et al. 1992. Long-term frontal brain metabolic changes in cocaine abusers. Synapse. 11:184-190.

30. Adinoff, B., et al. 2001. Limbic responsiveness to procaine in cocaineaddicted subjects. Am. J. Psychiatry. 158:390-398.

31. Volkow, N.D., et al. 1991. Changes in brain glucose metabolism in cocaine dependence and withdrawal. Am. J. Psychiatry. 148:621-626.

32. Volkow, N.D., et al. 1999. Association of methylphenidate-induced craving with changes in right striato-orbitofrontal metabolism in cocaine abusers: implications in addiction. Am. J. Psychiatry. 156:19-26.

33. Brody, A.L. 2002. Brain metabolic changes during cigarette craving. Arch. Gen. Psychiatry. 59:1162-1172.

34. Insel, T.R. 1992. Towards a neuroanatomy of obsessive-compulsive disorder. Arch. Gen. Psychiatry. 49:739-744.

35. Volkow, N.D., et al. 1993. Decreased dopamine D2 receptor availability is associated with reduced frontal metabolism in cocaine abusers. Synapse. 14:169-177.

36. Volkow, N.D., et al. 2001. Low level of brain dopamine $\mathrm{D}(2)$ receptors in methamphetamine abusers: association with metabolism in the orbitofrontal cortex. Am. J. Psychiatry. 158:2015-2021.

37. White, N.M. 1996. Addictive drugs as reinforcers: multiple partial actions on memory systems. Addiction. 91:921-949.

38. Kirk, J.M., Doty, P., and De Wit, H. 1998. Effects of expectancies on subjective responses to oral delta9-tetrahydrocannabinol. Pharmacol. Biochem. Behav. 59:287-293. 
39. Grant, S., et al. 1996. Activation of memory circuits during cue-elicited cocaine craving. Proc. Natl. Acad. Sci. U. S. A. 93:12040-12045.

40. Childress, A.R., et al. 1999. Limbic activation during cue-induced cocaine craving. Am. J. Psychiatry. 156:11-18.

41. Kilts, C.D., et al. 2001. Neural activity related to drug craving in cocaine addiction. Arch. Gen. Psychiatry. 58:334-341.

42. Wang, G.-J., et al. 1999. Regional brain metabolic activation during craving elicited by recall of previous drug experiences. Life Sci. 64:775-784

43. Letchworth, S.R., Nader, M.A., Smith, H.R., Friedman, D.P., and Porrino, L.J. 2001. Progression of changes in dopamine transporter binding site density as a result of cocaine self-administration in rhesus monkeys. J. Neurosci. 21:2799-2807.

44. Ito, R., Dalley, J.W., Robbins, T.W., and Everitt, B.J. 2002. Dopamine release in the dorsal striatum during cocaine-seeking behavior under the control of a drug-associated cue. J. Neurosci. 22:6247-6253.

45. Goldstein, R.Z., and Volkow, N.D. 2002. Drug addiction and its underlying neurobiological basis: neuroimaging evidence for the involvement of the frontal cortex. Am. J. Psychiatry. 159:1642-1652.

46. Royall, D.R., et al. 2002. Executive control function: a review of its promise and challenges for clinical research. A report from the Committee on Research of the American Neuropsychiatric Association. J. Neuropsychiatry Clin. Neurosci. 14:377-405.

47. Bechara, A., and Damasio, H. 2002. Decision-making and addiction (part I): impaired activation of somatic states in substance dependent individuals when pondering decisions with negative future consequences. Neuropsychologia. 40:1675-1689.

48. Ernst, M., et al. 2003. Decision making in adolescents with behavior disorders and adults with substance abuse. Am. J. Psychiatry. 160:33-40.

49. Robinson, T.E., Gorny, G., Mitton, E., and Kolb, B. 2001. Cocaine selfadministration alters the morphology of dendrites and dendritic spines in the nucleus accumbens and neocortex. Synapse. 39:257-266.

50. Ernst, M., et al. 2001. Effect of nicotine on brain activation during performance of a working memory task. Proc. Natl. Acad. Sci. U. S. A. 98:4728-4733.
51. Rosenkranz, J.A., and Grace, A.A. 2001. Dopamine attenuates prefrontal cortical suppression of sensory inputs to the basolateral amygdala of rats. J. Neurosci. 21:4090-4103.

52. Volkow, N.D., et al. 1999. Prediction of reinforcing responses to psychostimulants in humans by brain dopamine D2 receptor levels. Am. J. Psychiatry. 156:1440-1443.

53. Volkow, N.D., et al. 2002. Brain DA D2 receptors predict reinforcing effects of stimulants in humans: replication study. Synapse. 46:79-82.

54. Thanos, P.K., et al. 2001. Overexpression of dopamine D2 receptors reduces alcohol self-administration. J. Neurochem. 78:1094-1103.

55. Papp, M., Klimek, V., and Willner, P. 1994. Parallel changes in dopamine D2 receptor binding in limbic forebrain associated with chronic mild stress-induced anhedonia and its reversal by imipramine. Psychopharmacology. 115:441-446.

56. Hill, S.Y., et al. 2001. Right amygdala volume in adolescent and young adult offspring from families at high risk for developing alcoholism. Biol. Psychiatry. 49:894-905.

57. Franklin, T.R., et al. 2002. Decreased gray matter concentration in the insular, orbitofrontal, cingulate, and temporal cortices of cocaine patients. Biol. Psychiatry. 51:134-142.

58. Sora, I., et al. 2001. Molecular mechanisms of cocaine reward: combined dopamine and serotonin transporter knockouts eliminate cocaine place preference. Proc. Natl. Acad. Sci. U. S. A. 98:5300-5305.

59. Kreek, M.J., LaForge, K.S., and Butelman, E. 2002. Pharmacotherapy of addictions. Nat. Rev. Drug Discov. 1:710-726.

60. Miller, G.M., Yatin, S.M., De La Garza, R., II, Goulet, M., and Madras, B.K. 2001. Cloning of dopamine, norepinephrine and serotonin transporters from monkey brain: relevance to cocaine sensitivity. Brain Res. Mol. Brain Res. 87:124-143.

61. Volkow, N.D., et al. 2001. Dopamine transporter losses in methamphetamine abusers are associated with psychomotor impairment. Am. J. Psychiatry. 158:377-382.

62. Fowler, J.S., et al. 1996. Inhibition of monoamine oxidase B in the brains of smokers. Nature. 379:733-738. 